arXiv version: fonts, pagination and layout may vary from GTM published version

\title{
Filtering the fiber of the pinch map
}

\author{
BRAYTON GRAY
}

A new type of Hopf invariant is described for the fiber of the pinch map:

$$
F \longrightarrow X \cup C A \stackrel{\pi}{\longrightarrow} S A
$$

and this is used to study the boundary map in the fibration sequence of Cohen, Moore and Neisendorfer:

$$
\Omega^{2} S^{2 n+1} \stackrel{\partial_{n}}{\longrightarrow} \Omega F_{n} \longrightarrow \Omega P^{2 n+1} \longrightarrow \Omega S^{2 n+1} .
$$

The boundary map is shown to be compatible with the Hopf invariant and a filtration of the spliting is obtained.

55P99, 55Q20; 55Q25

\section{Introduction}

In [3], Cohen, Moore and Neisendorfer analyzed the homotopy type of the mod $p^{r}$ Moore space

$$
P^{2 n+1}=S^{2 n} \cup_{p^{r}} e^{2 n+1}
$$

by constructing a fibration sequence

$$
\Omega^{2} S^{2 n+1} \stackrel{\partial_{n}}{\longrightarrow} \Omega F_{n} \longrightarrow \Omega P^{2 n+1} \longrightarrow \Omega S^{2 n+1} .
$$

A key result is that both $\Omega F_{n}$ and $\Omega P^{2 n+1}$ contain a factor which is the loop space on a one point union of Moore spaces that are at least $4 n-2$ connected. Removing this factor (which can be inductively considered later) leaves a fibration sequence:

$$
\cdots \rightarrow \Omega^{2} S^{2 n+1} \stackrel{\bar{\partial}}{\longrightarrow} \bar{F}_{n} \longrightarrow T^{2 n+1} \longrightarrow \Omega S^{2 n+1}
$$

and they show that

$$
\bar{F}_{n} \cong S^{2 n-1} \times \prod_{k \geq 1} S^{2 n p^{k}-1}\left\{p^{r+1}\right\}
$$


An important remaining question is to understand the components of $\bar{\partial}$ :

$$
\begin{aligned}
& \bar{\partial}_{0}: \Omega^{2} S^{2 n+1} \longrightarrow S^{2 n-1} \\
& \bar{\partial}_{k}: \Omega^{2} S^{2 n+1} \longrightarrow S^{2 n p^{k}-1}\left\{p^{r+1}\right\} .
\end{aligned}
$$

$\bar{\partial}_{0}$ is well understood and plays a key role in homotopy theory. This is the noted "Cohen-Moore-Neisendorfer map". It fits into a homotopy commutative diagram:

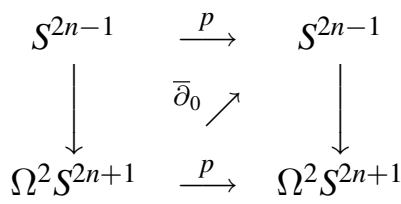

the consequence of which is that

$$
p \cdot \pi_{k}\left(S^{2 n+1}\right) \subset E^{2} \pi_{k-2}\left(S^{2 n-1}\right) \quad k>0
$$

from which the exponent result follows:

$$
p^{n} \pi_{k}\left(S^{2 n+1}\right)=0 \quad k>0 .
$$

There is much interest in understanding $\bar{\partial}_{k}$ for $k>0$; in particular, it is not known whether they are all null homotopic (see Anick and Gray [1], Cohen, Moore and Neisendorfer [3], Gray [5, 6] and Gray and Theriault [7]). In case $r>1$, Neisendorfer $[10,11]$ has shown that $\bar{\partial}_{k}=0$ for $k \geq 1$, so our emphasis will be on the case $r=1$.

A remarkable observation of Cohen, Moore and Neisendorfer [2] is that there is an isomorphism of Hopf algebras (see Appendix A):

$$
H_{*}\left(\Omega^{2} S^{2 n+1} ; \mathbb{Z} / p\right) \cong H_{*}\left(\bar{F}_{n} ; \mathbb{Z} / p\right) .
$$

However, the induced homomorphism $(\bar{\partial})_{*}=0$.

The intention of this work is to consider constructions involving $\bar{F}_{n}$ which are analogous to constructions involving $\Omega^{2} S^{2 n+1}$. We will be able to do this compatibly with the map $\bar{\partial}$. In particular, we will look at the filtration of Selick [12] and the classifying space of the double suspension [5]. Our results will follow from the construction of a new "Hopf invariant" type map:

$$
F_{n} \stackrel{h}{\longrightarrow} F_{n p}
$$

which induces an epimorphism in $p$-local homology. Recall that there is a $p$-local fibration sequence:

$$
S_{\left(p^{s}-1\right)}^{2 n} \longrightarrow S_{\infty}^{2 n} \stackrel{H_{p^{s}}}{\longrightarrow} S_{\infty}^{2 n p^{s}} .
$$


Here we are using James' notation $X_{\infty}$ for the reduced product space and $X_{(r)}$ for the subspace of words of length less than or equal to $r$. We also write $F_{(r)}$ for the analogous filtration of the relative James construction for $F$ (Gray [4]). By analogy, we compare $F_{\left(p^{s}-1\right)}$ to the fiber $\Delta_{s}$ of the Hopf invariant $h^{s}$ :

$$
\Delta_{s} \longrightarrow F_{n} \stackrel{h^{s}}{\longrightarrow} F_{n p^{s}}
$$

1.1 Theorem $\Omega \Delta_{s} \simeq \Omega F_{\left(p^{s}-1\right)} \times \Omega X_{s}$ where $X_{s}$ is a wedge of mod $p^{r}$ Moore spaces.

This will appear as Theorem 8.1. These same ideas lead to:

1.2 Theorem If $k>1$, there is a homotopy commutative diagram:

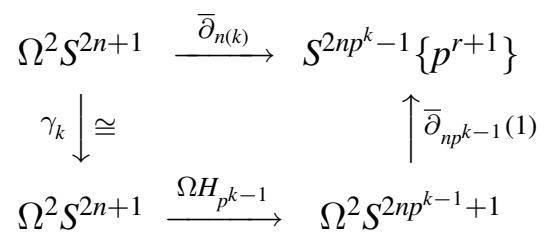

where $H_{p^{k}}$ is a James-Hopf invariant and $\gamma_{k}$ is a homotopy equivalence.

1.3 Theorem The map $\bar{\partial}_{n(k)}: \Omega^{2} S^{2 n+1} \longrightarrow S^{2 n p^{k}-1}\left\{p^{r+1}\right\}$ is homotopic to a composition:

$$
\Omega^{2} S^{2 n+1} \stackrel{\nu}{\longrightarrow} B W_{n} \stackrel{\epsilon}{\longrightarrow} S^{2 n p^{k}-1}\left\{p^{r+1}\right\} .
$$

These results follow from equation (9.2) and Corollary 10.3 respectively, in the sequel.

Throughout this paper all spaces will be assumed to be localized at a fixed prime $p>2$ and all homology will be $p$-local unless otherwise indicated.

\section{Filtration of $\Omega^{2} S^{2 n+1}$}

$H_{*}\left(\Omega^{2} S^{2 n+1} ; \mathbb{Z} / p\right)$ is a free commutative algebra on generators of dimensions $2 n p^{i}-1$, $2 n p^{i+1}-2$ for $i \geq 0$. Selick [12] has described a sequence of $H$-spaces whose homology filters $H_{*}\left(\Omega^{2} S^{2 n+1} ; \mathbb{Z} / p\right)$ by successively adding one generator at each stage. Let us write $S_{(k)}^{2 n}=J_{k}\left(S^{2 n}\right)$ for the $k$ th filtration of the James construction $J\left(S^{2 n}\right)=S_{\infty}^{2 n}$. Then the space capturing the first $2 s$ generators of $H_{*}\left(\Omega^{2} S^{2 n+1} ; \mathbb{Z} / p\right)$ is precisely 
$\Omega S_{\left(p^{s}-1\right)}^{2 n}$. Selick [12] defines spaces $G_{s}$ which contain the first $2 s+1$ generators. We define $G_{s}$ by a diagram of fibrations:

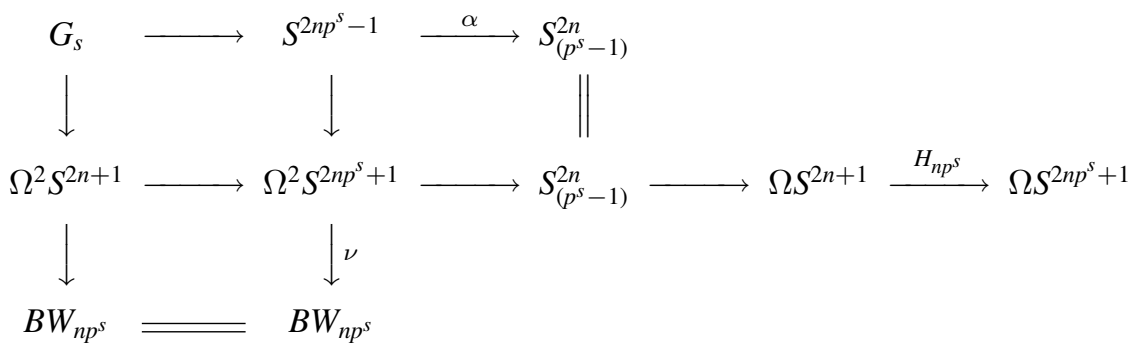

where $\alpha: S^{2 n p^{s}-1} \rightarrow S_{\left(p^{s}-1\right)}^{2 n}$ is the attaching map for the $2 n p^{s}$ cell of $S_{\infty}^{2 n}, H_{n p^{s}}$ is the appropriate James-Hopf invariant, and $B W_{n p^{s}}$ is the classifying space for the double suspension. Since $G_{s}$ is the fiber of $\nu \circ \Omega H_{n p^{s}}$, it is an $H$ space if $p>3$ (Gray [5, Proposition 6]). $G_{s}$ could also be described as the fiber of a Toda-Hopf invariant $\Omega S_{\left(p^{s+1}-1\right)}^{2 n} \stackrel{H^{\prime}}{\longrightarrow} \Omega S^{2 n p^{s+1}-1}$ using the techniques of Gray [5] and Moore and Neisendorfer [9]).

Constructing an analogous filtration for $\bar{F}_{n}$ is not difficult. The key result is in the compatibility of the two filtrations.

\section{Proof of Theorem CMN}

In this section we give a brief summary of the proof of the main result of Cohen, Moore and Neisendorfer [3].

Theorem CMN There is a diagram of fibration sequences:

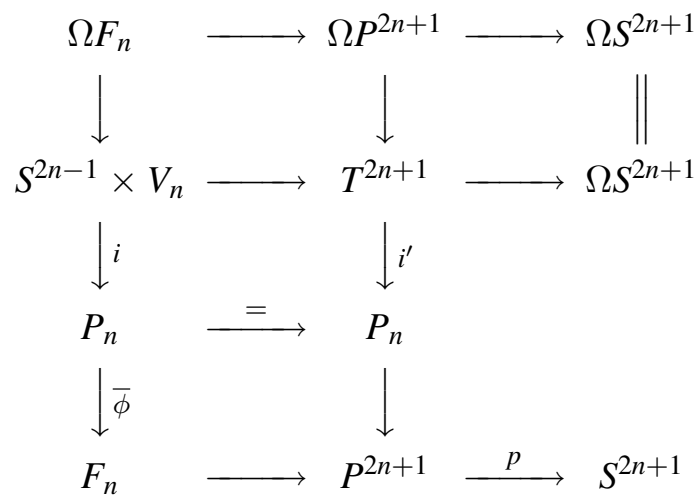

Geometry \& Topology Monographs 13 (2008) 
where the maps $i$ and $i^{\prime}$ are null homotopic, $p$ is the pinch map, $P_{n}$ is a one point union of mod $p^{r}$ Moore spaces of dimension $\geq 4 n$ and

$$
V_{n}=\prod_{k \geq 1} S^{2 n p^{k}-1}\left\{p^{r+1}\right\}
$$

Here $S^{m}\{d\}$ is the fiber of the degree $d$ map on $S^{m}$. The inessentiality of $i$ and $i^{\prime}$ implies that

$$
\begin{aligned}
& \Omega F_{n} \simeq S^{2 n-1} \times V_{n} \times \Omega P_{n} \\
& \Omega P^{2 n+1} \simeq T^{2 n+1} \times \Omega P_{n} .
\end{aligned}
$$

Sketch of the proof Both the mod $p^{r}$ homotopy and the mod $p$ homology of $\Omega P^{2 n+1}$ have differential Lie algebra structures, and the Hurewicz map is a Lie algebra homomorphism. Furthermore $\Omega F_{n}$ has an extended ideal structure. These structures are obtained from the Samelson product and the $r$ th Bockstein $\beta^{(r)}\left(\beta^{(i)}=0\right.$ for $\left.i<r\right)$. $H_{*}\left(\Omega P^{2 n+1} ; \mathbb{Z} / p\right)=U(L)$ where $L$ is a free Lie algebra on $v \in H_{2 n}\left(\Omega P^{2 n+1} ; \mathbb{Z} / p\right)$ and $u=\beta^{(r)}(v)$ and $U(L)$ is the universal enveloping algebra. $L^{(0)} \subset L$ is the Lie ideal generated by $x_{i}=a d^{i-1}(v)(u)$ for $i \geq 1$ and $H_{*}\left(\Omega F_{n} ; \mathbb{Z} / p\right)=U\left(L^{(0)}\right)$.

Furthermore the suspensions of the $x_{i}$,

$$
\sigma\left(x_{i}\right) \in H_{2 n i}\left(F_{n} ; \mathbb{Z} / p\right) \cong \mathbb{Z} / p
$$

are non zero. $u$ and $v$ lie in the image of the mod $p^{r}$ Hurewicz homomorphism, so all of $L$ and hence $L^{(0)}$ does as well

$$
L^{(0)} \subset \operatorname{im}\left\{\pi_{*}\left(\Omega F_{n} ; \mathbb{Z} / p^{r}\right) \longrightarrow H_{*}\left(\Omega F_{n} ; \mathbb{Z} / p\right)\right\}
$$

using the extended ideal structure in homotopy.

Both in homotopy and homology, $\beta^{(r)}\left(x_{p^{k}}\right)=0$, so one may construct an extension:

$$
P^{2 n p^{k}-1}\left(p^{r+1}\right) \stackrel{\delta_{k}}{\longrightarrow} \Omega F_{n}
$$

of the $\bmod p^{r}$ homotopy class

$$
P^{2 n p^{k}-1} \longrightarrow \Omega F_{n}
$$

representing $x_{p^{k}}$ in homology. The only property of the maps $\delta_{k}$ that is needed is that their Hurewicz image is $x_{p^{k}}$. From these maps the authors construct

$$
S^{2 n p^{k}-1}\left\{p^{r+1}\right\} \stackrel{e}{\longrightarrow} \Omega P^{2 n p^{k}}\left(p^{r+1}\right) \stackrel{\Omega \bar{\delta}_{k}}{\longrightarrow} \Omega F_{n}
$$


where $\bar{\delta}_{k}$ is the adjoint of $\delta_{k}$ and $e$ is a particular map described in the next section. These maps are assembled via loop multiplication to obtain:

$$
\Theta: S^{2 n-1} \times V_{n} \longrightarrow \Omega F_{n}
$$

The Bockstein $\beta^{(r)}$ is trivial in $H_{*}\left(S^{2 n-1} \times V_{n} ; \mathbb{Z} / p\right)$ but not in $H_{*}\left(\Omega F_{n} ; \mathbb{Z} / p\right)$. However $\Theta$ induces an isomorphism in the Bockstein homology of these homology groups (see Appendix A for a calculation of the Bockstein homology).

Next the authors construct a sequence of sub-Lie algebras $L^{(k+1)} \subset L^{(k)} \subset L^{(0)}$ via short exact sequences of Lie algebras:

$$
\begin{aligned}
& 0 \longrightarrow L^{(1)} \longrightarrow L^{(0)} \longrightarrow\left\langle\tau_{0}\right\rangle \quad \longrightarrow 0 \\
& 0 \longrightarrow L^{(k+1)} \longrightarrow L^{(k)} \longrightarrow\left\langle\tau_{k}, \sigma_{k}\right\rangle \longrightarrow 0 .
\end{aligned}
$$

Here $\left\langle\tau_{0}\right\rangle$ and $\left\langle\tau_{k_{1}} \sigma_{k}\right\rangle$ are free commutative Lie algebras generated by $\tau_{k}=x_{p^{k}}$ of dimension $2 n p^{k}-1$ and

$$
\sigma_{k}=\frac{1}{2 p} \sum_{i=1}^{p^{k}-1}\left(\begin{array}{c}
p^{k} \\
i
\end{array}\right)\left[x_{i}, x_{p^{k}-i}\right] \epsilon L^{(0)} .
$$

This is possible since $\tau_{k}$ is a generator and $\sigma_{k}$ is decomposable in $L^{(k-1)}$ but not in $L^{(k)}$ since $\frac{1}{2 p}\left(p_{p^{k-1}}^{p^{k}}\right)$ is a $p$-local unit and $x_{p^{k-1}}=\tau_{k-1} \notin L^{(k)}$.

It follows that there is a split short exact sequence of differential Hopf algebras:

$$
0 \longrightarrow U\left(L^{(k+1)}\right) \longrightarrow U\left(L^{(k)}\right) \longrightarrow H_{*}\left(S^{2 n p^{k}-1}\left\{p^{r+1}\right\} ; \mathbb{Z} / p\right) \rightarrow 0
$$

and hence

$$
U\left(L^{(\infty)}\right) \otimes H_{p}\left(S^{2 n-1} \times V_{n} ; \mathbb{Z} / p\right) \cong U\left(L^{(0)}\right)=H_{*}\left(\Omega F_{n} ; \mathbb{Z} / p\right)
$$

where $L^{(\infty)}=\bigcap_{k \geq 0} L^{(k)}$.

Consequently $H_{t}\left(U\left(L^{(\infty)}\right) ; \beta^{(r)}\right)=0$ for $t>0$ and hence $L^{(\infty)}$ has a basis consisting of classes $\left\{x_{\alpha}, \beta^{(r)} x_{\alpha}\right\}$. Since $x_{\alpha} \in L^{(\infty)} \subset L^{(0)}$ one can choose maps

$$
\phi_{\alpha}: P^{n_{\alpha}} \longrightarrow \Omega F_{n}
$$

whose homology image is $x_{\alpha}$ and $\beta^{(r)} x_{\alpha}$ using the extended ideal structure. Assembling these one produces a one point union of $\bmod p^{r}$ Moore spaces, $P_{n}$ and a map:

$$
\bar{\phi}: P_{n} \longrightarrow F_{n}
$$

such that the homology image of

$$
(\Omega \bar{\phi})_{*}: H_{*}\left(\Omega P_{n} ; \mathbb{Z} / p\right) \longrightarrow H_{*}\left(\Omega F_{n} ; \mathbb{Z} / p\right)
$$


is exactly $U\left(L^{(\infty)}\right)$. From this the authors produce a homotopy equivalence

$$
S^{2 n-1} \times V_{n} \times \Omega P_{n} \longrightarrow \Omega F_{n}
$$

by multiplying the maps $\Theta$ and $\bar{\phi}$ via the $H$ space structure in $\Omega F_{n}$. Let $\bar{F}_{n}$ be the fiber of $\bar{\phi}$. Then

$$
S^{2 n-1} \times V_{n} \stackrel{\Theta}{\longrightarrow} \Omega F_{n} \stackrel{\partial}{\longrightarrow} \bar{F}_{n}
$$

is a homotopy equivalence and $i$ is inessential. This completes the outline of the proof.

\section{Combinatorial description of $F_{n}$}

In [4] a combinatorial description of the fiber of the pinch map:

$$
F \longrightarrow X \cup C A \stackrel{\pi}{\longrightarrow} S A
$$

was given in the spirit of the James construction $X_{\infty}$ for $\Omega S X$. The model, designated $(X, A)_{\infty}$ consists of all words in $X_{\infty}$ with the property that all letters after the first letter are required to lie in $A$, where $A \subset X$. Alternatively, this can be described by a push out diagram:

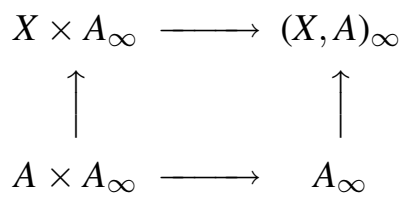

4.2 Proposition [4] There is a map $(X, A)_{\infty} \rightarrow F$ which is a homotopy equivalence when the inclusion $A \subset X$ is a cofibration.

There is an action of the monoid $A_{\infty}$ on $(X, A)_{\infty}$ and $(X, A)_{\infty}$ can be thought of as a universal space in the following sense. If $Y$ is any space on which $A_{\infty}$ acts and $g: X \rightarrow Y$ is any map such that $g(a)=a \cdot *$ for some point $* \in Y$, there is a unique $A_{\infty}$ equivariant map

$$
g_{\infty}:(X, A)_{\infty} \rightarrow Y
$$

(See $[4,4.2])$. The map $(X, A)_{\infty} \rightarrow F$ is constructed from the action $A_{\infty} \times F \rightarrow$ $\Omega S A \times F \rightarrow F$. 
The orbit space of $(X, A)_{\infty}$ under the action of $A_{\infty}$ is $X / A$ and we may use the universal property to establish the following diagram

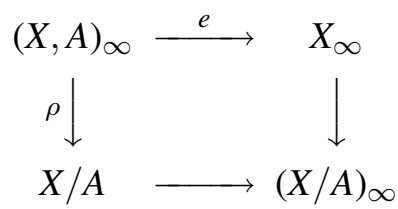

Note that the inclusion $e:(X / A)_{\infty} \rightarrow X_{\infty}$ is the unique $A_{\infty}$ equivariant extensions of the inclusion of $X$ in $X_{\infty}$.

Example 4.4 Let $A=S^{2 n-2} \subset P^{2 n-1}\left(p^{t}\right)=X$. Then $(X, A)_{\infty}$ is the homotopy fiber of the map of degree $p^{t}$

$$
(X, A)_{\infty}=S^{2 n-1}\left\{p^{t}\right\} \longrightarrow S^{2 n-1} \stackrel{p^{t}}{\longrightarrow} S^{2 n-1}
$$

and the map $e: S^{2 n-1}\left\{p^{t}\right\} \longrightarrow\left(P^{2 n-1}\left(p^{t}\right)\right)_{\infty} \cong \Omega P^{2 n}\left(p^{t}\right)$ is uniquely determined as a $\Omega S^{2 n-1}$ equivariant map extending the inclusion of $P^{2 n-1}\left(p^{t}\right)$.

One of the main features of the construction $(X, A)_{\infty}$ is that we can define functorial Hopf invariants using the same formulas as in James [8]. In particular we have a pointwise commutative diagram:

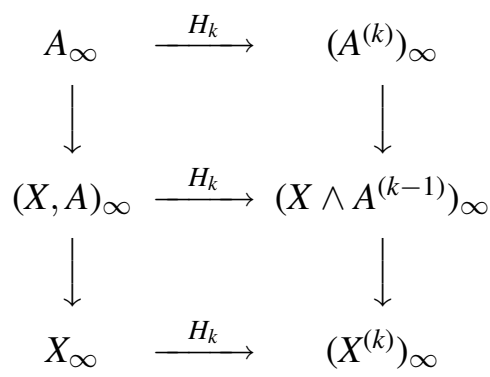

It would be desirable to construct functorial compressions of these maps:

$$
h:(X, A)_{\infty} \longrightarrow\left(X \wedge A^{(k-1)}, A^{(k)}\right)_{\infty} \subset\left(X \wedge A^{(k-1)}\right)_{\infty}
$$

but I have been unable to do this. In the next section we will construct a map $h$ of this form, but we have no knowledge of how it relates to $H_{k}$.

\section{Construction of $h: F_{n} \longrightarrow F_{n p}$}

In this section we will define a kind of Hopf invariant which is key for the results of this paper. 
5.1 Theorem Suppose $(X, A)$ is a suspension pair and $r \geq 1$. Then there is a map:

$$
h:(X, A)_{\infty} \longrightarrow\left(X \wedge A^{(r-1)}, A^{(r)}\right)_{\infty}
$$

such that the composition:

$$
X \times A_{\infty} \longrightarrow(X, A)_{\infty} \stackrel{h}{\longrightarrow}\left(X \wedge A^{(r-1)}, A^{(r)}\right)_{\infty} \stackrel{\rho}{\longrightarrow} X \wedge A^{(r-1)} / A^{(r)}
$$

is homotopic to the composition:

$$
X \times A_{\infty} \stackrel{1 \times H_{r-1}}{\longrightarrow} X \times A_{\infty}^{(r-1)} \longrightarrow X \wedge A_{\infty}^{(r-1)} \stackrel{\alpha}{\longrightarrow} X \wedge A^{(r-1)} \longrightarrow X \wedge A^{(r-1)} / A^{(r)}
$$

where $H_{r-1}: A_{\infty} \rightarrow A_{\infty}^{(r-1)}$ is any map and $\alpha$ uses the suspension structure of $X$ to collapse $X \wedge A_{\infty}^{(r-1)}$ to $X \wedge A^{(r-1)}$.

5.2 Corollary Let $F_{n}$ be the fiber of the pinch map

$$
p: P^{2 n+1}\left(p^{r}\right) \longrightarrow S^{2 n+1}
$$

for each $n$ and $H_{r-1}$ be any choice of James-Hopf invariants. Then there is a map:

$$
h: F_{n} \longrightarrow F_{r n}
$$

such that the composition:

$$
S^{2 n} \times \Omega S^{2 n+1} \longrightarrow F_{n} \stackrel{h}{\longrightarrow} F_{r n} \longrightarrow P^{2 r n+1}
$$

is homotopic to:

$$
S^{2 n} \times \Omega S^{2 n+1} \stackrel{1 \times H_{r-1}}{\longrightarrow} S^{2 n} \times \Omega S^{2(r-1) n+1} \longrightarrow S^{2 r n} \longrightarrow P^{2 r n+1}
$$

in particular, $h_{*}: H_{2 r n}\left(F_{n}\right) \longrightarrow H_{2 r n}\left(F_{r n}\right)$ is an isomorphism.

Note It is an easy calculation to see that

$$
H_{i}\left(F_{n}\right)=\left\{\begin{array}{cc}
\mathbb{Z}_{(p)} & n \mid i \\
0 & n \nmid i
\end{array}\right.
$$

and the map $F_{n} \stackrel{\pi}{\longrightarrow} P^{2 n+1}\left(p^{r}\right)$ is reduction $\bmod p^{r}$ in homology. In particular we have defined a map:

$$
F_{n} \longrightarrow P^{2 n r+1}\left(p^{r}\right)
$$

for each $r \geq 1$ which is onto in $p$-local homology. 
Proof of Theorem 5.1 We begin by constructing a map

$$
\mu:(X, A)_{\infty} \longrightarrow(X, A)_{\infty} / A_{\infty} \longrightarrow X \times A_{\infty} / A \rtimes A_{\infty} \equiv X \rtimes A_{\infty} / A \rtimes A_{\infty}
$$

which follows from the push out diagram (4.0). This map is functorial and we have a commutative diagram:

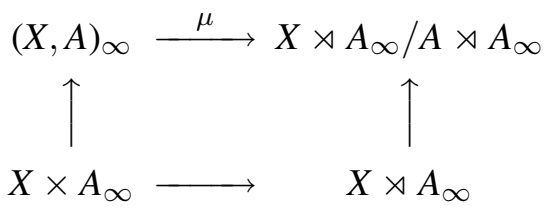

using the functorial property, we have the commutative diagram:

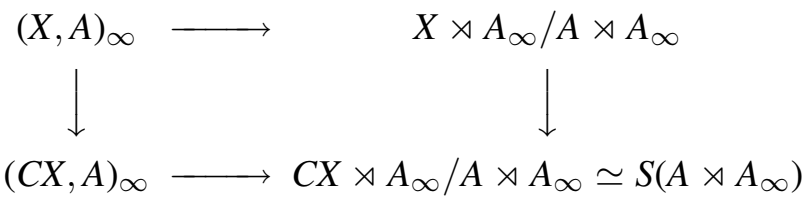

however $(C X, A)_{\infty}$ is the fiber of $C X / A \longrightarrow S A$ and hence is contractible. Consequently there is a lifting $\gamma$ of $\mu$ :

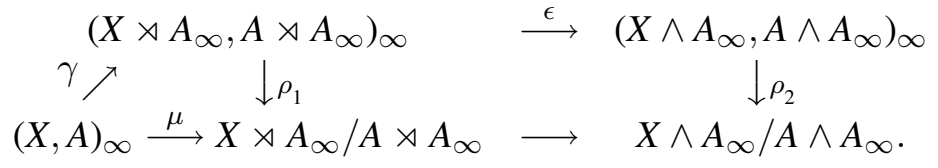

We now apply the homotopy commutative diagram:

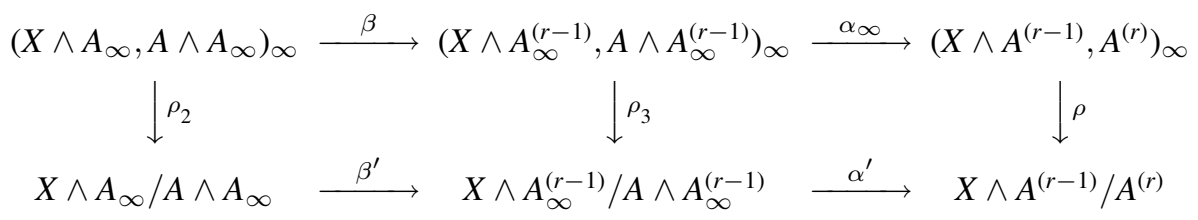

where $\beta$ and $\beta^{\prime}$ are defined using $H_{r-1}: A_{\infty} \rightarrow A_{\infty}^{(r-1)}$. We now define $h=\alpha_{\infty} \beta \epsilon \gamma$. Clearly the composition:

$$
X \times A_{\infty} \longrightarrow(X, A)_{\infty} \stackrel{h}{\longrightarrow}\left(X \wedge A^{(r-1)}, A^{(r)}\right)_{\infty} \stackrel{\rho}{\longrightarrow} X \wedge A^{(r-1)} / A^{(r)}
$$

is homotopic to the composition on the left and top in the diagram:

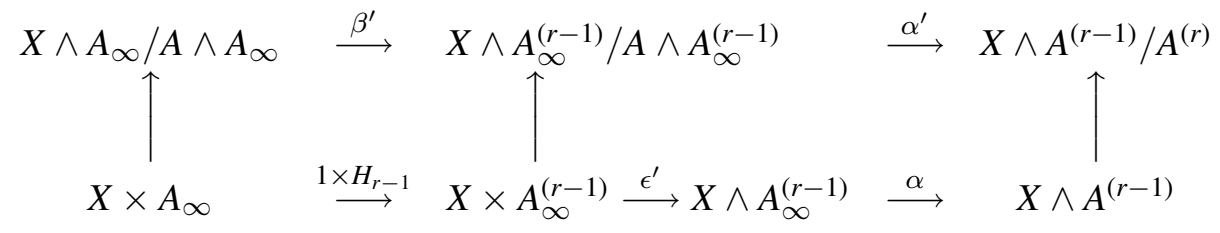

and consequently to the composite along the bottom and the right as well. 
5.3 Proposition The map $h: F_{n} \longrightarrow F_{n p^{s}}$ induces an epimorphism in $p$-local homology.

Proof We use the map $\Omega S^{2 n+1} \stackrel{\partial}{\longrightarrow} F_{n}$ which has degree $p$ in $H^{2 n k}$ for each $k>0$ to calculate the cup product structure in $H^{*}\left(F_{n}\right)$. We choose generators $e_{i} \in H^{2 n i}\left(F_{n}\right)$ such that $\partial^{*}\left(e_{i}\right)$ is $p$ times the generator of $H^{2 n i}\left(\Omega S^{2 n+1}\right)$ dual to the $i$ th power of a homology generator in $H_{2 n}\left(\Omega S^{2 n+1}\right)$. Then it is easy to see that

$$
e_{i} e_{j}=p\left(\begin{array}{c}
i+j \\
i
\end{array}\right) e_{i+j}
$$

Let us designate $d_{i} \in H^{2 n i p^{s}}\left(F_{n p^{s}}\right)$ for the corresponding generator; then

$$
h^{*}\left(d_{1}\right)=u_{1} e_{p^{s}}
$$

for some $p$ local unit $u_{1}$ by Corollary 5.2. We show that

$$
h^{*}\left(d_{i}\right)=u_{i} e_{i p^{s}}
$$

where $u_{i}$ is a $p$-local unit for each $i \geq 1$ by induction. Using the product structure we have

$$
\operatorname{pid}_{i}=d_{1} d_{i-1},
$$

so $\operatorname{pih}^{*}\left(d_{i}\right)=h^{*}\left(d_{1} d_{i-1}\right)=h^{*}\left(d_{1}\right) h^{*}\left(d_{i-1}\right)$

$$
\begin{aligned}
& =\left(u_{1} e_{p^{s}}\right)\left(u_{i-1} e_{(i-1) p^{s}}\right) \\
& =p u_{1} u_{i-1}\left(\begin{array}{c}
i p^{s} \\
p^{s}
\end{array}\right) e_{i p^{s}}
\end{aligned}
$$

It suffices to show that $\frac{1}{i}\left(\begin{array}{c}i p^{s} \\ p^{s}\end{array}\right)$ is a $p$-local unit.

Now let $v_{p}(m)$ be the exponent of $p$ in $m$ and $[x]$ be the greatest integer less than or equal to $x$. Then

$$
v_{p}(n !)=\sum_{i \geq 1}\left[\frac{n}{p^{i}}\right] .
$$

Consequently $v_{p}\left(\left(p^{s} i\right) !\right)=p^{s-1} i+p^{s-2} i+\cdots+i+v_{p}(i !)$ so

$$
\begin{aligned}
v_{p}\left(\left(\begin{array}{c}
p^{s} i \\
p^{s}
\end{array}\right)\right) & =v_{p}\left(\left(p^{s} i\right) !\right)-v_{p}\left(\left(p^{s}(i-1)\right) !\right)-v_{p}\left(p^{s} !\right) \\
& =v_{p}(i !)-v_{p}((i-1) !) \\
& =v_{p}(i) .
\end{aligned}
$$

and we are done. 
By Corollary 5.2, the composition

$$
\Omega S^{2 n+1} \stackrel{\partial}{\longrightarrow} F_{n} \stackrel{h}{\longrightarrow} F_{n p^{s}} \longrightarrow P^{2 n p^{2}+1}
$$

is null homotopic, so there is a lifting $\widetilde{H}_{p^{s}}$

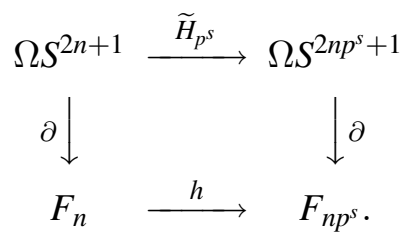

5.4 Proposition $\left(\widetilde{H}_{p^{s}}\right)_{*}: H_{*}\left(\Omega S^{2 n+1}\right) \rightarrow H_{*}\left(\Omega S^{2 n p^{s}+1}\right)$ is an epimorphism.

This follows since both maps labelled $\partial$ have degree $p$ in homology and $h_{*}$ is an epimorphism.

In particular, the fiber of $\widetilde{H}_{p^{s}}$ is $S_{\left(p^{s}-1\right)}^{2 n}$ as if $\widetilde{H}_{p^{s}}$ were the James-Hopf invariant $H_{p^{s}}$. Both $\Omega \widetilde{H}_{p^{s}}$ and $\Omega H_{p^{s}}$ can be placed in the fibration sequence induced by the inclusion $S_{\left(p^{s}-1\right)}^{2 n} \subset \Omega S^{2 n+1}$, so there is an equivalence $\gamma_{s}: \Omega^{2} S^{2 n+1} \rightarrow \Omega^{2} S^{2 n+1}$ such that

$$
\Omega \widetilde{H}_{p^{s}}=\Omega H_{p^{s}} \circ \gamma_{s}
$$

hence we have:

5.5 Corollary There is a homotopy commutative diagram

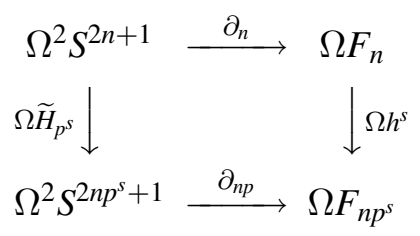

where $\Omega \widetilde{H}_{p^{s}} \sim \Omega H_{p^{s}} \circ \gamma_{s}$ for some homotopy equivalence $\gamma_{s}: \Omega^{2} S^{2 n+1} \longrightarrow$ $\Omega^{2} S^{2 n+1}$.

\section{A Filtered Decomposition}

Let $\Delta_{s}$ be the homotopy fiber of $h^{s}: F_{n} \rightarrow F_{n p^{s}}$. In this section we will compare the decompositions of $\Omega F_{n}$ and $\Omega F_{n p^{s}}$ and prove

Geometry \& Topology Monographs 13 (2008) 
6.1 Theorem $\Omega \Delta_{s} \cong S^{2 n-1} \times \prod_{1 \leq k<s} S^{2 n p^{k}-1}\left\{p^{r+1}\right\} \times \Omega S^{2 n p^{s}-1} \times \Omega R_{s}$ where $R_{s}$ is a wedge of $\bmod p^{r}$ Moore spaces.

Let $\Psi: S^{2 n-1} \times V_{n} \rightarrow S^{2 n p-1} \times V_{n p}$ be defined by first projecting onto $V_{n}$ and then applying

$$
V_{n}=S^{2 n p-1}\left\{p^{2}\right\} \times V_{n p} \stackrel{\rho \times 1}{\longrightarrow} S^{2 n p-1} \times V_{n p}
$$

where $\rho: S^{2 n p-1}\left\{p^{2}\right\} \rightarrow S^{2 n p-1}$ is the projection.

6.2 Lemma With appropriate choices of the maps $\Theta_{n}$ and $\Theta_{n p}$ there is a homotopy commutative diagram:

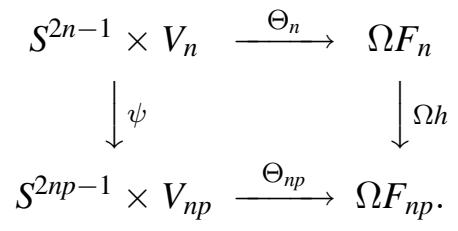

Proof Given choices $\bar{\delta}_{k}: P^{2 n p^{k}}\left(p^{r+1}\right) \rightarrow F_{n}$ inducing epimorphisms in $p$-local cohomology, define $\bar{\delta}_{k}(n p)$ as the composition:

$$
P^{2 n p^{k+1}}\left(p^{r+1}\right) \stackrel{\bar{\delta}_{k+1}}{\longrightarrow} F_{n} \stackrel{h}{\longrightarrow} F_{n p}
$$

From this we construct

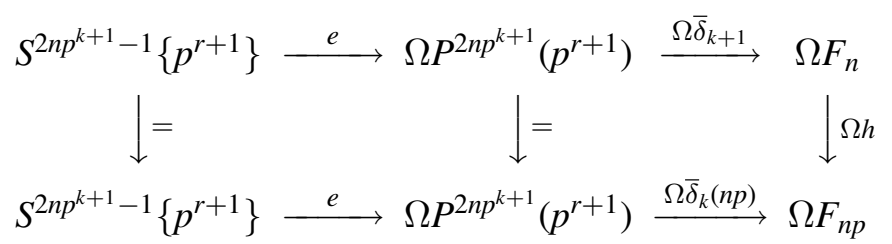

where $k>0$, and

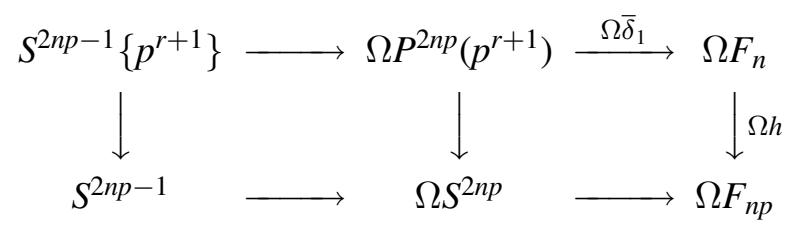

using diagram (4.3). Multiplying these together in order gives the result.

Geometry \& Topology Monographs 13 (2008) 
6.3 Lemma The map $\bar{\phi}_{n}: P_{n} \rightarrow F_{n}$ can be chosen so that $P_{n}=P_{n p} \vee Q_{n}$ and there is a homotopy commutative diagram:

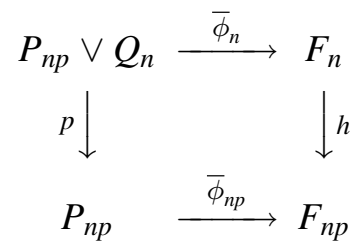

where $p$ is the projection.

Proof Since $h: H_{*}\left(F_{n} ; \mathbb{Z} / p\right) \rightarrow H_{*}\left(F_{n p} ; \mathbb{Z} / p\right)$ is onto the same holds for

$$
U\left(L^{(0)}(n)\right) \cong H_{*}\left(\Omega F_{n} ; \mathbb{Z} / p\right) \rightarrow H_{*}\left(\Omega F_{n p} ; \mathbb{Z} / p\right) \cong U\left(L^{(0)}(n p)\right)
$$

in fact the generators $x_{i p} \in L^{(0)}(n)$ satisfy

$$
h_{*}\left(x_{i p}(n)\right)=u_{i} x_{i}(n p)
$$

where $u_{i}$ is a $p$-local unit (see Proposition 5.3).

Now given a basis $\left\{x_{\alpha}, \beta^{(r)} x_{\alpha}\right\}$ for $L^{(\infty)}(n p)$, each $x_{\alpha}$ is a Lie bracket in the $x_{i}(n p)$ and this element consequently lifts to the same bracket in $x_{i p}(n)$. Thus these liftings are linearly independent and can be chosen as part of a basis. They are all in the image of the mod $p^{r}$ Hurewicz homomorphism. Thus we have chosen generators for $L^{(\infty)}(n)$ which sort into those which are lifting of the generators for $L^{(\infty)}(n p)$ and the others. Realizing these via the Hurewicz homomorphism gives the maps $\bar{\phi}_{n}$. By a change in basis for $Q_{n}$, we can assume that the map $p: P_{n} \rightarrow P_{n p}$ is trivial on $Q_{n}$.

We now use Lemma 6.3 to construct the following ladder of fibrations:

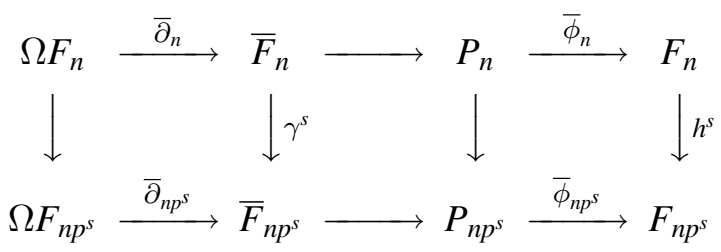

and we use Lemma 6.2 to construct compatible equivalences:

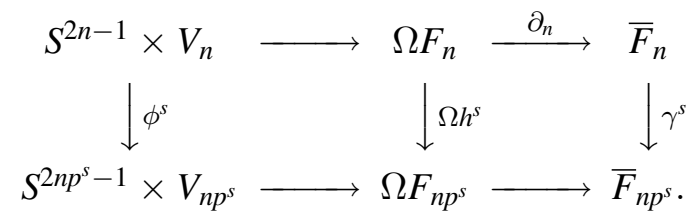

Geometry \& Topology Monographs 13 (2008) 
Taking fibers vertically in (6.4), we have a fibration sequence:

$$
\Omega \Delta_{s} \rightarrow K_{s} \rightarrow R_{s} \rightarrow \Delta_{s}
$$

where $R_{s}=\left(Q_{n} \vee \cdots \vee Q_{p n^{s}-1}\right) \rtimes \Omega P_{n p^{s}}$ is a wedge of mod $p^{r}$ Moore spaces and

$$
K_{s}=S^{2 n-1} \times \prod_{1 \leq k<s} S^{2 n p^{k}-1}\left\{p^{r+1}\right\} \times \Omega S^{2 n p^{s}-1} .
$$

We use the splitting in (6.5) to construct a splitting

$$
K_{s} \rightarrow \Omega \Delta_{s} \rightarrow K_{s}
$$

This completes the proof.

\section{Decomposition of $\Omega F_{\left(p^{s}-1\right)}$}

Let $F_{\left(p^{s}-1\right)}$ be the $2 n p^{s}-1$ skeleton of $F$; since the mod $p^{r}$ homotopy classes

$$
P^{2 n i-1} \longrightarrow \Omega F_{n}
$$

representing $x_{i}$ in homology factor through $F_{\left(p^{s}-1\right)}$ when $i<p^{s}$, the suspension map

$$
\sigma: \widetilde{H}_{*}\left(\Omega F_{\left(p^{s}-1\right)} ; Z / p\right) \longrightarrow H_{*}\left(F_{\left(p^{s}-1\right)} ; k\right)
$$

is onto. By Proposition A.3 of the Appendix, $H_{*}\left(\Omega F_{\left(p^{s}-1\right)} ; \mathbb{Z} / p\right)$ is a tensor algebra on classes $x_{i}$ of dimension $2 n i-1$ for $i<p^{s}$.

7.1 Theorem $\Omega F_{\left(p^{s}-1\right)} \simeq S^{2 n-1} \times \prod_{1 \leq k<s} S^{2 n p^{k}-1}\left\{p^{r+1}\right\} \times \Omega S^{2 n p^{s}-1} \times \Omega Q_{s}$ where $Q_{s} \subset Q_{s+1} \subset \cdots \subset P_{n}$ is a wedge of $\bmod p^{r}$ Moore spaces for some choice of $P_{n}$ in [3].

Proof This proceeds along the same lines as [3]. The maps

$$
\bar{\delta}_{k}: P^{2 n p^{k}} \rightarrow F
$$

factor through $F_{\left(p^{s}-1\right)}$ when $k<s$ as does the restriction

$$
\left.\bar{\delta}_{s}\right|_{S^{2 n p^{s}-1}}: S^{2 n p^{s}-1} \rightarrow F
$$

We thus construct a map

$$
\Theta_{s}: K_{s}=S^{2 n-1} \times \prod_{1 \leq k<s} S^{2 n p^{k}-1}\left\{p^{r+1}\right\} \times \Omega S^{2 n p^{s}-1} \rightarrow \Omega F_{\left(p^{s}-1\right)}
$$


as before. The Bocksteins $\beta^{(i)}$ for $i<r$ are trivial and $\Theta_{s}$ induces an isomorphism in the homology under the $r$ th Bockstein. To see this observe that the Bockstein homology spectral sequence of $H_{*}\left(\Omega F_{\left(p^{s}-1\right)} ; \mathbb{Z} / p\right)$ is a restriction of the spectral sequence for $H_{*}(\Omega F ; \mathbb{Z} / p)$ (see Appendix A). $H_{*}\left(\Omega F_{\left(p^{s}-1\right)} ; \mathbb{Z} / p\right)=U\left(L_{S}^{(0)}\right)$ where $L_{s}^{(0)}$ is a free Lie algebra generated by $x_{i}$ of dimension $2 n i-1$ for $1 \leq i<p^{s}$.

To complete the proof we construct Lie algebras $L_{s}^{(k)} \subset L_{s}^{(0)}$ which are compatible with the subalgebras $L^{(k)}$ of $L^{(0)}$. First we examine the construction of $L^{(k)}$. (See Section 2). Recall that we have short exact sequences:

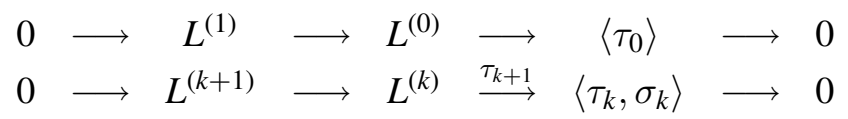

where $\pi_{k+1}$ is any map of Lie algebras such that

$$
\begin{aligned}
& \pi_{k+1}\left(\sigma_{k}\right)=\sigma_{k} \\
& \pi_{k+1}\left(\tau_{k}\right)=\tau_{k}
\end{aligned}
$$

where $\sigma_{k}, \tau_{k} \in L^{(k)} \subset L^{(0)}$ are given by the formulas:

$$
\begin{aligned}
\tau_{k} & =x_{p^{k}} \\
\sigma_{k} & =\frac{1}{2 p} \sum_{i=1}^{p^{k}-1}\left(\begin{array}{c}
p^{k} \\
i
\end{array}\right)\left[x_{i}, x_{p^{k}-i}\right]
\end{aligned}
$$

Nothing is said about the value of $\pi_{k+1}$ on the other generators. We need to be more specific at this point. Let us define the weight of an element in a free Lie algebra to be the minimal number of brackets in any term; in particular, $L^{(0)}$ is free on generators $x_{i}$, we define $\omega\left(x_{i}\right)=1, \omega[x, y]=\omega(x)+\omega(y)$ and $\omega\left(\Sigma a_{i}\right)=\min \omega\left(a_{i}\right)$. For an element $z \in L^{(k)}$ we define the weight of $z$ to be the weight considered as an element of $L^{(0)}$. Thus $\omega\left(\tau_{k}\right)=1$ and $\omega\left(\sigma_{k}\right)=2$. We further specify the Lie algebra homomorphism $\pi_{k+1}$ by demanding that $\pi_{k+1}(z)=0$ if $\omega(z)>2$. Now define $L_{s}^{(k)}=L_{s} \cap L^{(k)}$ for 
$k \leq s$. Since $\sigma_{k}, \tau_{k} \in L_{s}^{(k)}$, we have short exact sequences:

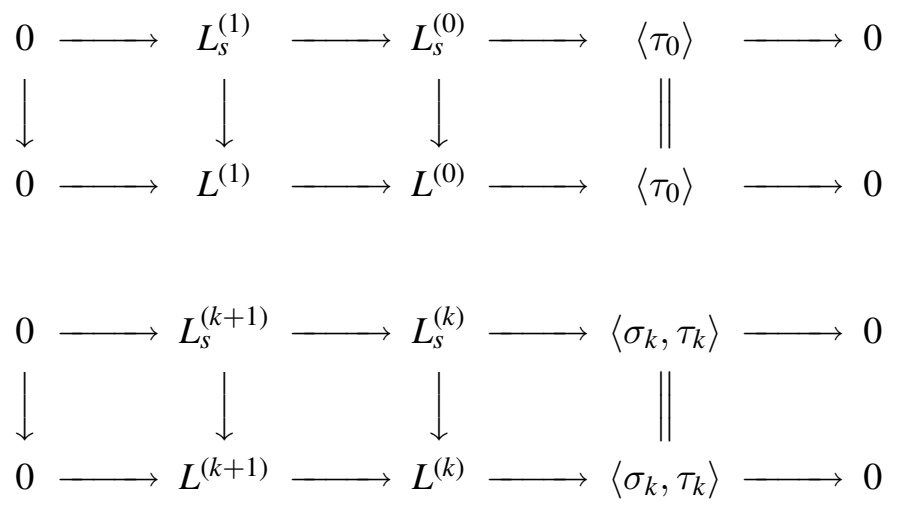

for $k<s$.

Define $L_{s}^{(s+1)}$ to be the kernel of $L_{s}^{(s)} \rightarrow\left\langle\sigma_{s}\right\rangle \rightarrow 0$. In fact we have $L_{s}^{(s+1)} \subset L^{(\infty)}$. To see this we need to show that $\pi_{r+1}\left(L_{s}^{(s+1)}\right)=0$ for $r \geq s$. The generators of $L_{s}^{(s+1)}$ of filtration 1 are of the form $x_{i}$ for $i<p^{s}$ and those of filtration 2 are of the form $\left[x_{i}, x_{j}\right]$ for $i, j<p^{s}$. None of these have dimension $2 n p^{s}-1$, so $L_{s}^{(s+1)}$ lies in the kernel of the composition $L^{(s+1)} \rightarrow\left\langle\tau_{s}, \sigma_{s}\right\rangle \rightarrow\left\langle\tau_{s}\right\rangle$. Consequently $L_{s}^{(s+1)} \subset L^{(s+1)}$. Similarly for $r>s$ the generators of $L_{s}^{(s+1)}$ of weight 1 and 2 have dimensions $\leq 4 n\left(p^{s}-1\right)$ and consequently their images are 0 in $\left\langle\sigma_{r}, \tau_{r}\right\rangle$ for $r>s$.

It follows that we may first choose a basis for $L_{s}^{(s+1)}$ and then choose a basis for $L^{(\infty)}$ containing these elements. This completes the proof.

7.2 Corollary There is a commutative diagram of fibration sequences:

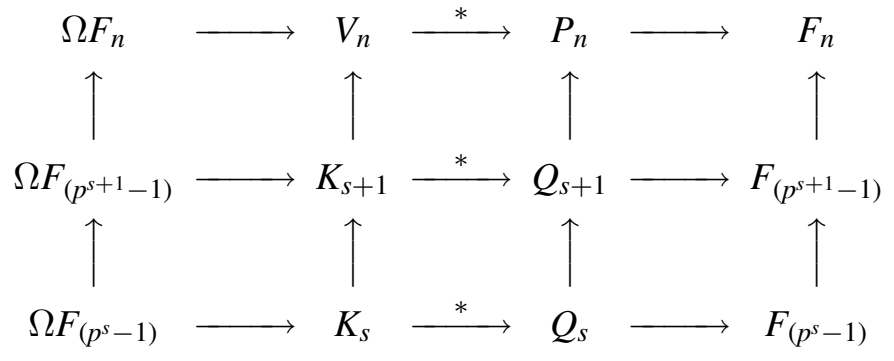

where all the vertical maps are mod $p$ homology monomorphisms and each $Q_{s}$ is a wedge of $\bmod p^{r}$ Moore spaces. 


\section{On the sequence $F_{\left(p^{s}-1\right)} \rightarrow F_{n} \rightarrow F_{n p^{s}}$}

Recall that there is a $p$-local fibration sequence

$$
S_{\left(p^{s}-1\right)}^{2 n} \rightarrow S_{\infty}^{2 n} \stackrel{H_{p^{s}}}{\longrightarrow} S_{\infty}^{2 n p^{s}}
$$

By analogy, we compare $F_{\left(p^{s}-1\right)}$ to the fiber $\Delta_{s}$ of the Hopf invariant $h^{s}$ :

$$
\Delta_{s} \rightarrow F_{n} \stackrel{h^{s}}{\longrightarrow} F_{n p^{s}}
$$

8.1 Theorem $\Omega \Delta_{s} \simeq \Omega F_{\left(p^{s}-1\right)} \times \Omega X_{s}$ where $X_{s}$ is a wedge of mod $p^{r}$ Moore spaces.

Proof For dimensional reasons, the inclusion $F_{\left(p^{s}-1\right)} \subset F_{n}$ lifts to $\Delta_{s}$. Consider the pull back diagram:

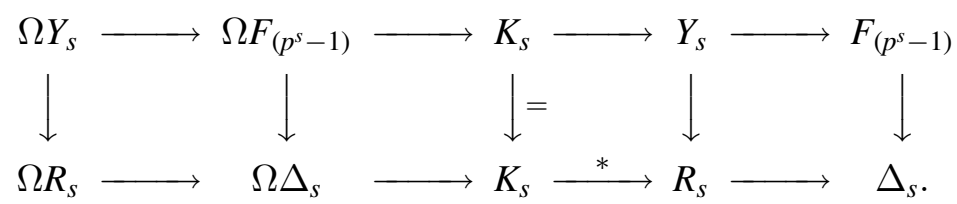

The map $K_{s} \rightarrow \Omega \Delta_{s} \rightarrow \Omega F_{n}$ constructed in Section 6 is obtained from maps:

$$
\begin{array}{lllll}
\bar{\delta}_{k}: P^{2 n p^{k}} & \longrightarrow & F_{n} & & k<s \\
S^{2 n p^{s}-1} & \longrightarrow & P^{2 n p^{s}} \quad \stackrel{\bar{\delta}_{s}}{\longrightarrow} F_{n} &
\end{array}
$$

and all of these maps factor through $F_{\left(p^{s}-1\right)}$ for dimensional reasons. Consequently the retraction $K_{s} \rightarrow \Omega \Delta_{s}$ factors through $\Omega F_{\left(p^{s}-1\right)}$ as well and hence the map $K_{s} \rightarrow Y_{s}$ is null homotopic. Consequently $\Omega Q_{s} \simeq \Omega Y_{s}$ and the map $Q_{s} \rightarrow F_{\left(p^{s}-1\right)}$ lifts to an equivalence $Q_{s} \simeq Y_{s}$. Now the inclusion $Q_{s} \rightarrow P_{n}$ factors through $R_{s}$, so $R_{s}=Q_{s} \vee E_{s}$ and we have a diagram of fibrations:

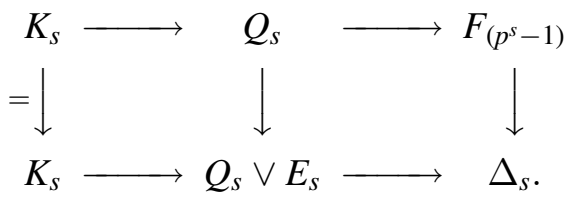

Now the fiber of $Q_{s} \rightarrow Q_{s} \vee E_{s}$ is $\Omega\left(E_{s} \rtimes \Omega Q_{s}\right)$ which is a retract of $\Omega\left(Q_{s} \vee E_{s}\right)=\Omega R_{s}$ and hence a retract of $\Omega \Delta_{s}$. This completes the proof with $X_{s}=E_{s} \rtimes \Omega Q_{s}$. 


\section{Factorization of $\bar{\partial}$}

In this section we will consider the components of

$$
\bar{\partial}_{n}: \Omega^{2} S^{2 n+1} \longrightarrow S^{2 n-1} \times V_{n}=S^{2 n-1} \times \prod_{k \geq 1} S^{2 n p^{k}-1}\left\{p^{r+1}\right\}
$$

we will write $\partial_{n}(k): \Omega^{2} S^{2 n+1} \longrightarrow S^{2 n p^{k}-1}\left\{p^{r+1}\right\}$ for the $k$ th component, $k>0$ and $\bar{\partial}_{n}(0)$ for the projection $\Omega^{2} S^{2 n+1} \longrightarrow S^{2 n-1}$.

We begin by combining the diagram from Corollary 5.5 :

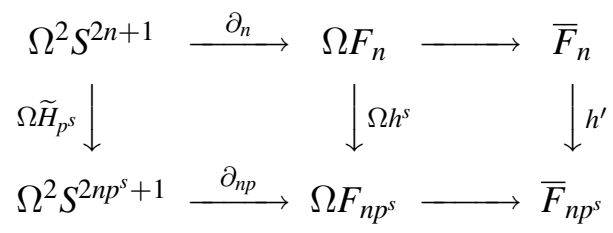

with the equivalences from Lemma 6.2

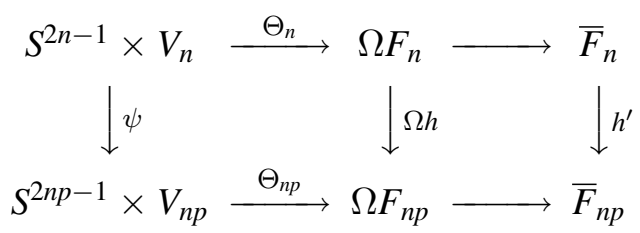

where $h^{\prime}$ is defined in Lemma 6.3, to get a homotopy commutative diagram:

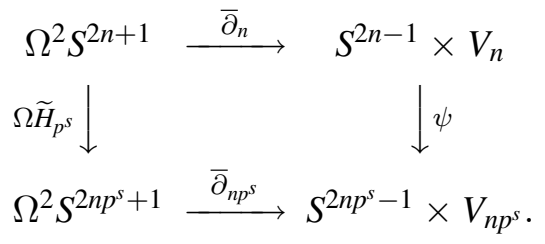

From this we see that

$$
\bar{\partial}_{n}(k+1)=\bar{\partial}_{n p^{k}}(1) \circ \Omega H_{p^{k}} \circ \gamma_{k}, \quad k \geq 0
$$

while

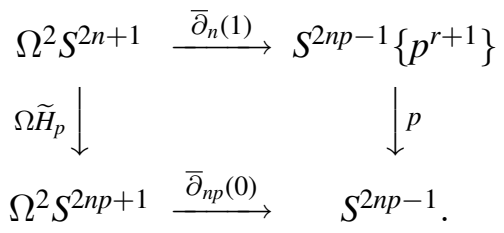

Putting these together gives:

$$
\bar{\partial}_{n}=\bar{\partial}_{n}(0)+\sum_{k=0}^{\infty} \bar{\partial}_{n p^{k-1}}(1) \circ \Omega H_{p^{k-1}} \circ \gamma_{k}
$$


where $\gamma_{k}: \Omega^{2} S^{2 n+1} \rightarrow \Omega^{2} S^{2 n+1}$ is an equivalence. Equation (9.2) proves Theorem 1.2.

Remark If we had better control over the relationship between the Hopf invariants $h^{s}$ and the James-Hopf invariant, we could remove the factor $\gamma_{k}$.

9.3 Proposition Suppose $\bar{\partial}_{n}(1): \Omega^{2} S^{2 n+1} \rightarrow S^{2 n p-1}\left\{p^{r+1}\right\}$ is null homotopic for each $n$. Then

(a) $\bar{\partial}_{n}$ factors through $S^{2 n-1}$

(b) The loops on the pth James-Hopf invariant:

$$
\Omega H_{p}: \Omega^{2} S^{2 n+1} \longrightarrow \Omega^{2} S^{2 n p+1}
$$

is homotopic to a composition:

$$
\Omega^{2} S^{2 n+1} \longrightarrow \Omega^{2} P^{2 n p+1} \stackrel{\Omega^{2} p}{\longrightarrow} \Omega^{2} S^{2 n p+1}
$$

(c) The fiber $B$ of $\bar{\partial}_{n p}(0): \Omega^{2} S^{2 n p+1} \rightarrow S^{2 n p-1}$ is a retract of $\Omega^{2} P^{2 n p+1}$

(d) $B \simeq B W_{n}$.

Proof (a) follows directly from (9.2). To verify (b), note that by hypothesis

$$
\Omega H_{p}: \Omega^{2} S^{2 n+1} \longrightarrow \Omega^{2} S^{2 n p+1}
$$

lifts so the fiber $B$ of $\bar{\partial}_{n p}(0)$. The following diagram provides a map from $B$ to $\Omega^{2} P^{2 n p+1}$ :

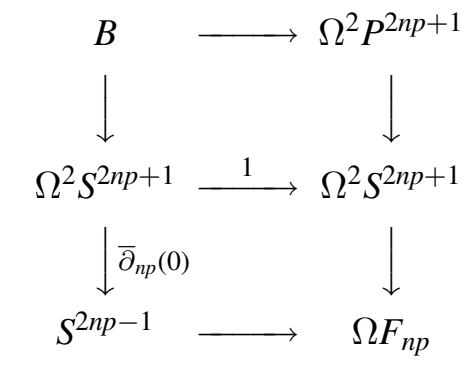

(c) follows since $\Omega F_{n} \simeq S^{2 n p-1} \times V_{n p} \times \Omega P_{n p}$, so

$$
\Omega^{2} P^{2 n p+1} \simeq B \times \Omega V_{n p} \times \Omega^{2} P_{n p} .
$$

To verify (d), note that $S^{2} B W_{n}$ is a retract of $S^{2} \Omega^{2} S^{2 n+1}$ by [5], so there is a map $S^{2} B W_{n} \rightarrow P^{2 n p+1}$ which is non zero in homology, It's adjoint provides an equivalence:

$$
B W_{n} \rightarrow \Omega^{2} P^{2 n p+1} \rightarrow B .
$$




\section{Relationship with $B W_{n}$}

In this section we describe a factorization of $\bar{\partial}$ using the classifying space $B W_{n}$ for the double suspension. Recall from [5]

10.1 Proposition Suppose $\alpha: S^{2 n} \rightarrow Y$ and $\beta: S^{2 n-1} Y \rightarrow S^{4 n-1}$ are maps such that $\beta \circ S^{2 n-1} \alpha: S^{4 n-1} \rightarrow S^{2 n-1} Y \rightarrow S^{4 n-1}$ is homotopic to the identity. Then there is a fibration sequence:

$$
S^{2 n-1} \stackrel{\bar{\alpha}}{\longrightarrow} \Omega Y \longrightarrow B .
$$

This is natural in the following sense: Suppose we are also given

$$
\begin{aligned}
& \alpha^{\prime}: S^{2 n} \longrightarrow Z \\
& \beta^{\prime}: S^{2 n-1} Z \longrightarrow S^{4 n-1} \\
& f: Y \longrightarrow Z
\end{aligned}
$$

such that $\beta \sim \beta^{\prime} \circ S^{2 n-1} f$ and $\alpha^{\prime} \sim f \alpha$.

Then we have a homotopy commutative diagram:

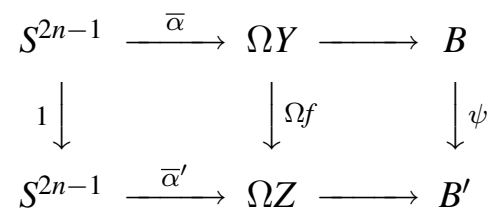

10.2 Corollary There is a homotopy commutative diagram:

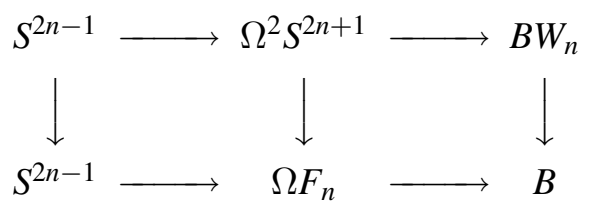

Since $S^{2 n-1}$ is a retract of $\Omega F_{n}, B \simeq V_{n} \times \Omega P_{n}$ and we have:

10.3 Corollary There is a homotopy commutative diagram:

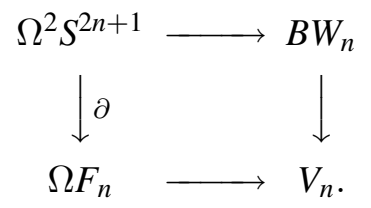

In particular, $\bar{\partial}_{n}(k): \Omega^{2} S^{2 n+1} \rightarrow S^{2 n p^{k}-1}\left\{p^{r+1}\right\}$ factors through $B W_{n}$, proving Theorem 1.3. 


\section{A Appendix}

This appendix has two purposes. We begin with a discussion of an observation that appears in the work of Cohen, Moore and Neisendorfer. We repeat it here because it sheds light on their results and is easily extended to the case of the filtrations in Section 7. In addition, we prove a general result about the homology of certain loop spaces which does not seem to be in the literature.

We begin by looking at the homology Serre spectral sequence for the fibering

$$
\Omega F_{n} \longrightarrow \Omega P^{2 n+1} \stackrel{\Omega \pi}{\longrightarrow} \Omega S^{2 n+1} .
$$

This is a multiplicative spectral sequence with

$$
E_{p, q}^{2} \cong H_{p}\left(\Omega S^{2 n+1} ; \mathbb{Z} / p\right) \otimes H_{q}\left(\Omega F_{n} ; \mathbb{Z} / p\right) .
$$

Since all elements of $E_{p, 0}^{2}$ are permanent cycles, $E^{2}=E^{\infty}$. Restricting this fibration to $S_{(k)}^{2 n}$ leads to a fibration

$$
\Omega F_{n} \longrightarrow E_{k} \longrightarrow S_{(k)}^{2 n}
$$

and $H_{*}\left(E_{k} ; \mathbb{Z} / p\right) \subset H_{*}\left(E_{k+1} ; \mathbb{Z} / p\right) \subset \cdots \subset H_{*}\left(\Omega P^{2 n+1} ; \mathbb{Z} / p\right)$.

Thus $H_{*}\left(\Omega P^{2 n+1} ; \mathbb{Z} / p\right)$ is a filtered differential group using $\beta^{(r)}$. It has a spectral sequence:

$$
E_{p, q}^{2}=H_{p}\left(\Omega S^{2 n+1} ; \beta^{(r)}\right) \otimes H_{q}\left(H_{*}\left(\Omega F_{n} ; \mathbb{Z} / p\right), \beta^{(r)}\right)
$$

converging to $H_{p+q}\left(\Omega P^{2 n+1} ; \mathbb{Z} / p\right)=0$ if $p+q>0$. This gives a multiplicative spectral sequence:

$$
E_{p, q}^{2}=\mathbb{Z}_{p}\left[u_{2 n}\right] \otimes H_{q}\left(H_{*}\left(\Omega F_{n} ; \mathbb{Z} / p\right) ; \beta^{(r)}\right) \Rightarrow 0 .
$$

This spectral sequence has the same form as the Serre Spectral sequence for the homology of the fibration:

$$
\Omega^{2} S^{2 n+1} \longrightarrow P \Omega S^{2 n+1} \longrightarrow \Omega S^{2 n+1} .
$$

The differentials are forced by the multiplicative structure and consequently these spectral sequences are isomorphic. From this we conclude

A.1 Proposition [3] $H_{*}\left(H_{*}\left(\Omega F_{n} ; \mathbb{Z} / p\right) ; \beta^{(r)}\right) \cong H^{*}\left(\Omega^{2} S^{2 n+1} ; \mathbb{Z} / p\right)$ as algebras and co-algebras.

A.2 Corollary $H_{*}\left(H_{*}\left(\Omega F_{\left(p^{s}-1\right)} ; \mathbb{Z} / p\right) ; \beta^{(r)}\right) \cong H_{*}\left(\Omega S_{\left(p^{s}-1\right)}^{2 n} ; \mathbb{Z} / p\right)$. 
The later follows by restricting the spectral sequence to the first $p^{s}-1$ columns.

A.3 Proposition Suppose that $X$ is simply connected and $k$ is a field. Suppose that the suspension map:

$$
\sigma: \widetilde{H}_{r-1}(\Omega X ; k) \longrightarrow \widetilde{H}_{r}(X ; k)
$$

is onto. Choose a right inverse $\tau$ to $\sigma$ and let $T_{*} \subset \widetilde{H}_{*}(\Omega X ; k)$ be the image of $\tau$. Then $H_{*}(\Omega X ; k)$ is the tensor algebra on $T_{*}$.

Proof We will use the Serre spectral sequence for the loop space fibration. Note that it is a spectral sequence of $H_{*}(\Omega X ; k)$ modules. Observe that the suspension map can be factored

$$
\widetilde{H}_{r-1}(\Omega X ; k)=E_{0, r-1}^{2} \longrightarrow E_{0, r-1}^{r} \stackrel{d_{r}}{\cong} E_{r, 0}^{r} \longrightarrow E_{r, 0}^{2}=\widetilde{H}_{r}(X ; k)
$$

where the first homomorphism is onto and the last is $1-1$. By hypothesis, the total composite is onto so the last homomorphism is an isomorphism. In particular if $x \in E_{r, 0}^{2}$, $d_{i}(x)=0$ for $i<r$ and $d_{r}(x)=[\tau(x)] \in E_{0, r-1}^{\tau}$ which is non zero. Now define

$$
F(r)_{p}=\left\{\begin{array}{cl}
H_{p}(X ; k) & \text { if } p=0 \text { or } p \geq r \\
0 & \text { if } 0<p<r .
\end{array}\right.
$$

We claim that there is a quotient module $Q(r)$ of $H_{*}(\Omega X ; k)$ such that

$$
E_{p, q}^{r} \cong F(r)_{p} \otimes Q(r)_{q} .
$$

This is certainly true when $r=2$ with $Q(2)=H_{*}(\Omega X ; k)$. We proceed by induction. Because of this tensor product decomposition,

$$
d^{r}(f \otimes q)=d^{r}(f \otimes 1)(1 \otimes q)=1 \otimes \tau(f) q
$$

and hence $d_{p, q}^{r}=0$ unless $p=r$. That is, all non zero differentials terminate on the fiber. Furthermore

$$
d_{r, q}^{r}: E_{r, q}^{r} \longrightarrow E_{0, q+r-1}^{r}
$$

is a monomorphism since otherwise the kernel would need to be in the image of some differential. Consequently

$$
\begin{aligned}
\operatorname{ker} d_{r} & =F(r+1) \otimes Q(r) \\
\lim d_{r} & =F(r+1) \otimes T_{*} Q(r) .
\end{aligned}
$$

Now define $Q(r+1)=Q(r) / T_{*} Q(r)$ which completes the induction. 
We observe that the spectral sequence is completely determined by $\tau$. We complete the proof by applying the comparison theorem. Let $Y$ be a wedge of spheres together with an isomorphism:

$$
\phi: H_{*}(Y ; k) \longrightarrow H_{*}(X ; k) .
$$

We will define a map of spectral sequences:

$$
E_{p, q}^{r}(Y) \longrightarrow E_{p, q}^{r}(X)
$$

$\phi$ defines a isomorphism $F(\phi): F(r)(Y) \longrightarrow F(r)(X)$. Since $Y$ is a co-H space, there is a map $v: Y \longrightarrow S \Omega Y$; we define $\tau(Y)$ as the composite

$$
\widetilde{H}_{r}(Y ; k) \stackrel{v_{*}}{\longrightarrow} \widetilde{H}_{r}(S \Omega Y ; k) \cong \widetilde{H}_{r-1}(\Omega Y ; k) .
$$

Now define $\Theta: H_{*}(\Omega Y ; k) \longrightarrow H_{*}(\Omega X ; k)$ is the ring homomorphism determined by

$$
\Theta(\tau(Y)(x))=\tau(x)(\phi(x)) .
$$

This defines a $H_{*}(\Omega Y ; k)$ module homomorphism

$$
E_{p, q}^{2}(Y) \longrightarrow E_{p, q}^{2}(X)
$$

However at each stage

$$
d^{r}(f \otimes q)=\left\{\begin{array}{cc}
1 \otimes \tau(f) q & r=p \\
0 & r \neq p .
\end{array}\right.
$$

So this defines a map of spectral sequences. Since they both converge to 0 and $\phi$ is an isomorphism, $\Theta$ is as well completing the proof.

\section{References}

[1] D Anick, B Gray, Small H spaces related to Moore spaces, Topology 34 (1995) 859-881 MR1362790

[2] F R Cohen, J C Moore, J A Neisendorfer, Torsion in homotopy groups, Ann. of Math. (2) 109 (1979) 121-168 MR519355

[3] F R Cohen, J C Moore, J A Neisendorfer, Exponents in homotopy theory, from: "Algebraic topology and algebraic $K$-theory (Princeton, N.J., 1983)", Ann. of Math. Stud. 113, Princeton Univ. Press, Princeton, NJ (1987) 3-34 MR921471

[4] B Gray, On the homotopy groups of mapping cones, Proc. London Math. Soc. (3) 26 (1973) 497-520 MR0334198

[5] B Gray, On the iterated suspension, Topology 27 (1988) 301-310 MR963632 
[6] B Gray, EHP spectra and periodicity. I. Geometric constructions, Trans. Amer. Math. Soc. 340 (1993) 595-616 MR1152323

[7] B Gray, S Theriault, On the double suspension and the mod-p Moore space, from: “An alpine anthology of homotopy theory", Contemp. Math. 399, Amer. Math. Soc., Providence, RI (2006) 101-121 MR2222507

[8] IM James, Reduced product spaces, Ann. of Math. (2) 62 (1955) 170-197 MR0073181

[9] J C Moore, J A Neisendorfer, Equivalence of Toda-Hopf invariants, Israel J. Math. 66 (1989) 300-318 MR1017169

[10] J Neisendorfer, Product decompositions of the double loops on odd primary Moore spaces, Topology 38 (1999) 1293-1311 MR1690159

[11] J Neisendorfer, James-Hopf invariants, Anick's spaces, and the double loops on odd primary Moore spaces, Canad. Math. Bull. 43 (2000) 226-235 MR1754027

[12] P Selick, A spectral sequence concerning the double suspension, Invent. Math. 64 (1981) 15-24 MR621768

Dept of Math, Stats and Comp Sci (M/C 249), University of Illinois at Chicago

851 South Morgan Street, Chicago, IL 60607-7045, USA

brayton@uic.edu

Received: 24 July 2006 Revised: 19 January 2007 